\section{P249 ASSESSMENT OF HEALTH RELATED QUALITY OF LIFE (HROOL) IN NON-CYSTIC FIBROSIS BRONCHIECTASIS USING A NEW DISEASE-SPECIFIC TOOL FOR MEASURING HRQOL: THE QUALITY OF LIFE-BRONCHIECTASIS (QOL-B) QUESTIONNAIRE}

doi:10.1136/thoraxjnl-2011-201054c.249

\begin{abstract}
${ }^{1}$ A R McCullough, ${ }^{1} \mathrm{C}$ M Hughes, ${ }^{1} \mathrm{M}$ Tunney, ${ }^{2} \mathrm{~J} S$ Elborn, ${ }^{1} \mathrm{~J} \mathrm{M}$ Bradley. ${ }^{1}$ School of Pharmacy, Queen's University Belfast, Belfast, Northern Ireland; ${ }^{2}$ Centre for Infection and Immunity, Queen's University Belfast, Belfast, Northern Ireland
\end{abstract}

Introduction The QOL-B is the first disease-specific HRQoL questionnaire for bronchiectasis. Quittner et al (ATS, 2009) have provided preliminary data on reliability and validity of the questionnaire. It has not been used in bronchiectasis populations outside of the USA.

Aim To assess HRQoL using the disease-specific OOL-B questionnaire and explore the relationship between $\mathrm{FEV}_{1} \%$ predicted, age, gender, time from 1st Pseudomonas aeruginosa isolate and QOL-B.

Methods This study is part of a larger study exploring adherence to treatment in bronchiectasis. Patients with bronchiectasis (confirmed by HRCT) were recruited if they had a positive sputum culture for $P$ aeruginosa and were using nebulised antibiotics. Patients selfcompleted the QOL-B (eight domains, each scored 0-100, lowhighHRQoL). Spirometry was performed according to ATS/ERS guidelines. Stepwise multiple regression analyses were completed for each QOL-B domain using four independent variables: age, gender, $\mathrm{FEV}_{1} \%$ and time from 1 st $P$ aeruginosa isolate.

Results 71 patients were recruited: 22M/49F; mean (SD) age 65 (8) yrs; $\mathrm{FEV}_{1} 60$ (25) \% predicted; mean time since first $P$ aeruginosa isolate 51 (41) months. QOL-B domains showed impairment in HRQoL, mean (range): physical functioning 31 (0-100); vitality 37 (0-78); health perceptions 39 (8-92); social functioning 42 $(0-100)$; role functioning $46(0-100)$; respiratory functioning 53 (8-100); treatment burden 56 (11-89); and emotional functioning 73 (8-100). Males had significantly lower $(p=0.046)$ physical functioning than females, mean (SD) 22.8 (23) vs 36.58 (27) respectively; however gender did not explain the variance in any of the $\mathrm{QOL}-\mathrm{B}$ domains. Age, $\mathrm{FEV}_{1} \%$ and time from 1 st $P$ aeruginosa isolate together explained $5.5-26.9 \%$ of variance $\left(r^{2}\right)$ in domain scores. Age was related to health perceptions $\left(\mathrm{r}^{2}=12.1 \%\right)$, treatment burden $\left(r^{2}=11.5 \%\right)$, social $\left(r^{2}=15.6 \%\right)$, role $\left(r^{2}=10.7 \%\right)$ and respiratory $\left(\mathrm{r}^{2}=16 \%\right)$ functioning domains. $\mathrm{FEV}_{1} \%$ was related to physical $\left(\mathrm{r}^{2}=18.8 \%\right)$ and role $\left(\mathrm{r}^{2}=8.7 \%\right)$ functioning domains. Time from $1 \mathrm{st}$ $P$ aeruginosa isolate was related to vitality $\left(r^{2}=11 \%\right)$, physical $\left(\mathrm{r}^{2}=6.9 \%\right)$, social $\left(\mathrm{r}^{2}=5.6 \%\right)$ and emotional $\left(\mathrm{r}^{2}=5.5 \%\right)$ functioning domains.

Conclusion HRQoL is impaired in patients with bronchiectasis. Older age is associated with better HRQoL. Higher $\mathrm{FEV}_{1} \%$ is associated with better physical and role functioning. Vitality, physical, social and emotional functioning improves with increased time from 1 st $P$ aeruginosa isolate

\section{P250 MYCOPLASMA PNEUMONIA. PRESENTING FEATURES AND DIAGNOSIS IN OUR DISTRICT GENERAL HOSPITAL IN 2010}

\author{
doi:10.1136/thoraxjn|-2011-201054c.250
}

${ }^{1} \mathrm{~K}$ S Caldwell, ${ }^{1} \mathrm{R}$ Johns, ${ }^{2} \mathrm{~L}$ Teare, ${ }^{3} \mathrm{H}$ S Jenkins. ${ }^{1}$ Broomfield Hospital, Chelmsford, UK; ${ }^{2}$ Department of Microbiology, Broomfield Hospital, Chelmsford, UK; ${ }^{3}$ Department of Respiratory Medicine, Broomfield Hospital, Chelmsford, UK

Introduction Mycoplasma pneumoniae is a common cause of pneumonia. Incidence ranges from 0.5 to 5.0 per 1000 population or up to $20 \%$ of all pneumonias, and generally declines with age, being rare in adults over 50. Classically epidemics occur in 4 to 7-year cycles. Usual features are insidious onset "viral"-type symptoms, including fever, headache, dyspnoea and dry cough, together with a variety of extra pulmonary manifestations. Diagnosis is often missed because of the atypical and unusual presentation.

Methods and Results We examined electronic records of patients during 2010 diagnosed with $M$ pneumoniae by an elevated specific IgM immunoassay method. There were 35 cases. Of those, 20 required acute admission to our hospital (18 adults and two children). In adults, common presenting features were fever, cough, headaches, lethargy and myalgia. Major presenting features, however, were meningitis/encephalitis in two patients, StevensJohnson syndrome in 1, confusion in 1, and haemoptysis in 1. In six adults (33\%), the diagnosis was not made during hospital admission, and symptoms were erroneously attributed to presumptive diagnoses of viral meningitis, acute viral illness, dyspnoea of unknown cause, asthma/pericarditis, and an acute drug reaction. We compared length of admission in patients diagnosed early on in admission to those misdiagnosed or diagnosed late; early diagnosis of $M$ pneumoniae using this method was associated with significantly shorter lengths of stay.

Conclusion and Discussion An appreciation of common presenting clinical features of Mycoplasma is important in ensuring the diagnosis is made promptly and not missed. The advantage of an IgM based assay is the detection of early/acute illness rather than convalescent disease (as in the case of parallel assays of acute and convalescent samples), having the potential to change management, refine antibiotics where appropriate and also to potentiate early discharge

\section{P251 JUNIOR DOCTORS' INTERPRETATION OF CXRS IS MORE CONSISTENT THAN CONSULTANTS IN THE CONTEXT OF POSSIBLE PNEUMONIA}

doi:10.1136/thoraxjnl-2011-201054c.251

${ }^{1} \mathrm{~B}$ Singh, ${ }^{1} \mathrm{~J}$ Curtis, ${ }^{2} \mathrm{~S}$ B Gordon, ${ }^{3} \mathrm{P} J$ Diggle, ${ }^{1} \mathrm{D}$ G Wootton. ${ }^{1}$ University Hospital Aintree NHS Foundation Trust, Liverpool, UK; ${ }^{2}$ Liverpool School of Tropical Medicine, Department of Respiratory Infection, Liverpool, UK; ${ }^{3}$ University of Lancaster, CHICAS, School of Health and Medicine, Lancaster, UK

Introduction and Objectives BTS guidelines suggest the diagnostic test for pneumonia (in hospital) is a CXR. Since management should be commenced within $4 \mathrm{~h}$ of arrival the initial interpretation of the CXR is most often performed by junior doctors. Consultants review patients and their CXRs within $24 \mathrm{~h}$ of admission and a radiologist's report is issued at a later time point but diagnosis dependent decisions - whether to admit, risk stratification and whether to give antibiotics-fall to junior doctors. We measured the inter-observer agreement within groups of doctors involved at key points in CAP diagnosis and management.

Methods 24 admission CXRs from patients attending a large teaching hospital between February and April 2011 with a suspected diagnosis of CAP were collected on a radiology computer workstation. Eleven reporting radiologists, eight attending consultants and twenty-two junior doctors from a range of acute medical specialties were recruited. All 41 recruits independently interpreted the CXRs, answering "yes" or "no" for presence of pneumonic infiltrate. Agreement within groups was tested using the FleissCuzick extension of Cohen's $\kappa$ statistic (abbreviated here as $\kappa$ ).

Results Agreement ( $\kappa$ scores) for each group of doctors are shown in Abstract P251 figure 1. Radiologists had moderate agreement ( $\kappa 0.52$, CI 0.49 to 0.55 ), junior doctors had moderate agreement ( $\kappa 0.47, \mathrm{CI}$ 0.45 to 0.48 ) and attending consultants fair agreement $(\kappa 0.34, \mathrm{CI}$ 0.30 to 0.38 ). 


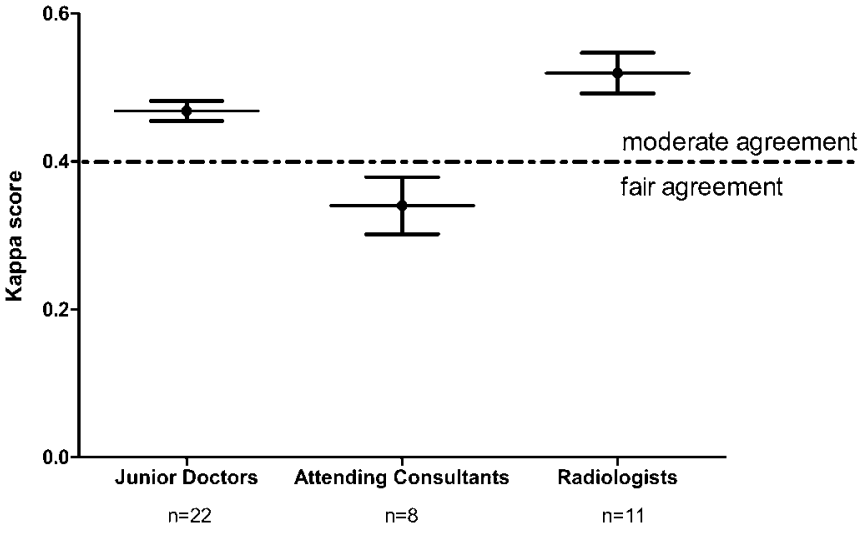

Abstract P251 Figure 1 Consistency between doctors when reporting CXRs in the context of possible CAP ( $\kappa$ with $95 \% \mathrm{Cl}$ ).

Conclusions In the context of possible pneumonia, the CXR was not consistently reported by any group. Junior doctors were more consistent than attending consultants and radiologists were most consistent. A possible explanation for these differences is that junior doctors, by necessity, have developed similar ideas of what they will consider pneumonic where as consultants who less frequently make the initial diagnostic decision vary in their criteria for diagnosing pneumonia. This study does not present a "gold standard" interpretation and therefore does not address the issue of accuracy but it does raise questions about to what extent the CXR ever be regarded as a "reliable" diagnostic test.

\section{COPD and drugs: new and old concepts P252 ONCE-DAILY NVA237 IMPROVES SYMPTOMS, AND REDUCES COPD EXACERBATIONS AND ASSOCIATED HOSPITALISATIONS: THE GLOW1 TRIAL}

\section{doi:10.1136/thoraxjnl-2011-201054c.252}

${ }^{1} \mathrm{~A}$ D'Urzo, ${ }^{2} \mathrm{~J}$ A van Noord, ${ }^{3} \mathrm{C}$ Martin, ${ }^{3} \mathrm{R}$ Horton, ${ }^{4} \mathrm{D}$ Banerij, ${ }^{4} Y \mathrm{Lu},{ }^{3} \mathrm{~V}$ K T Alagappan, ${ }^{3} \mathrm{~T}$ Overend. ${ }^{1}$ Department of Family and Community Medicine, University of Toronto, Ontario, Canada; ${ }^{2}$ Atrium Medisch Centrum, Heerlen, The Netherlands; ${ }^{3}$ Novartis Horsham Research Centre, Horsham, West Sussex, UK; ${ }^{4}$ Novartis Pharmaceuticals Corporation, East Hanover, New Jersey, USA

Introduction Symptoms profoundly impact daily life of COPD patients. We assessed the influence of the once-daily (qd) long-acting muscarinic antagonist (LAMA) NVA237 (glycopyrronium bromide) on symptoms and exacerbations in patients with moderate-tosevere COPD.

Methods Patients were randomised $(2: 1)$ to 26 weeks double-blind treatment with NVA237 $50 \mu \mathrm{g}$ qd or placebo (PBO) via a single-dose dry powder inhaler (Breezhaler ${ }^{\circledR}$ device). Efficacy was assessed by bronchodilation (trough $\mathrm{FEV}_{1}$ at Week 12), breathlessness on the transition dyspnoea index (TDI), HRQoL via the St. George's Respiratory Questionnaire (SGRQ), and rescue medication use. The effect on COPD exacerbations and related hospitalisations was also assessed.

Results 822 patients were randomised; $80.5 \%$ completed. NVA237 significantly increased total TDI focal score vs PBO at Week 26 (difference $1.04,95 \%$ CI 0.583 to $1.504 ; \mathrm{p}<0.0001$ ); exceeding the minimum clinically important difference ([MCID] $=1$ point). Significantly more patients achieved MCID in TDI score with NVA237 (61.3\% vs $48.3 \%$; OR $1.74,95 \%$ CI 1.249 to 2.415 ; $\mathrm{p}=0.001)$. SGRQ total score was significantly reduced with NVA237 $(-2.81 ; p=0.004) ; \%$ of patients achieving a clinically meaningful improvement in SGRQ (=4 point reduction) was significantly higher with NVA237 (56.8\% vs 46.3\%; OR 1.58, 95\% CI 1.138 to
2.196; $\mathrm{p}=0.006)$. NVA237 significantly reduced rescue medication use at Week 26 ( -0.46 puffs/day, $p=0.005)$. NVA237 significantly prolonged time to first moderate/severe COPD exacerbation by $31 \%$ (HR 0.69, 95\% CI 0.50 to $0.949 ; \mathrm{p}=0.023$ ) and time to first severe COPD exacerbation necessitating hospitalisation (HR $0.35,95 \% \mathrm{CI}$ 0.141 to $0.857 ; p=0.022$ ). NVA237 significantly reduced hospitalisations due to COPD exacerbation (OR 0.34; $\mathrm{p}=0.024$ ).

Conclusion Once-daily NVA237 provided significant improvements in dyspnoea and SGRQ total score, with lower rescue medication use, and reduced risk of exacerbation and associated hospitalisations vs $\mathrm{PBO}$.

\section{P253 NVA237 ONCE DAILY OFFERS RAPID AND CLINICALLY MEANINGFUL BRONCHODILATION IN COPD PATIENTS THAT IS MAINTAINED FOR 24 H: THE GLOW1 TRIAL}

doi:10.1136/thoraxjnl-2011-201054c.253

${ }^{1} \mathrm{~T}$ D'Urzo, ${ }^{2} \mathrm{G}$ Ferguson, ${ }^{3} \mathrm{C}$ Martin, ${ }^{3} \mathrm{~V}$ K T Alagappan, ${ }^{4} \mathrm{D}$ Banerji, ${ }^{4} \mathrm{Y}$ Lu, ${ }^{3} \mathrm{R}$ Horton, ${ }^{3} \mathrm{~T}$ Overend. ${ }^{1}$ Department of Family and Community Medicine, University of Toronto, Ontario, Canada; ${ }^{2}$ Pulmonary Research Institute of Southeast Michigan, Livonia, Michigan, USA; ${ }^{3}$ Novartis Horsham Research Centre, Horsham, West Sussex, UK; ${ }^{4}$ Novartis Pharmaceuticals Corporation, East Hanover, New Jersey, USA

Introduction NVA237 (glycopyrronium bromide) is an inhaled longacting muscarinic antagonist (LAMA) in development for the oncedaily (qd) treatment of COPD. The GLOW1 study evaluated the efficacy and safety of NVA237 in patients with moderate-to-severe COPD.

Methods Patients were randomised (2:1) to 26 weeks double-blind treatment with NVA237 $50 \mu \mathrm{g}$ qd or placebo (PBO). Study drugs were administered via a single-dose dry powder inhaler (Breezhaler ${ }^{\circledR}$ device). Primary efficacy endpoint: trough $\mathrm{FEV}_{1}$ (mean of $23 \mathrm{~h}$ $15 \mathrm{~min}$ and $23 \mathrm{~h} 45 \mathrm{~min}$ post-dose values) vs $\mathrm{PBO}$ after 12 weeks.

Results 822 patients were randomised; mean age was 63.9 years, mean post-bronchodilator $\mathrm{FEV}_{1}$ was $55 \%$ predicted. $80.5 \%$ completed the study. At Week 12 there was a statistically significant and clinically relevant difference between NVA237 vs PBO in mean trough $\mathrm{FEV}_{1}(108 \mathrm{ml} ; \mathrm{p}<0.001)$. Trough $\mathrm{FEV}_{1}$ was also significantly higher at Day 1 and Week 26 (treatment difference: $105 \mathrm{ml}$ and $113 \mathrm{ml}$, respectively; $\mathrm{p}<0.001$ ). Serial spirometry in a subpopulation of patients showed statistically superior $(p<0.001)$ and clinically meaningful improvements in $\mathrm{FEV}_{1}$ with NVA237 vs $\mathrm{PBO}$ at all timepoints on Day 1, Week 12 and Week 26. NVA237 had a rapid onset of action with an increased $\mathrm{FEV}_{1}$ of $93 \mathrm{ml}$ at $5 \mathrm{~min}$ and $144 \mathrm{ml}$ at $15 \mathrm{~min}$ vs $\mathrm{PBO}$ after the first dose on Day $1(\mathrm{p}<0.001)$. Overall, the incidence of adverse events (AEs) was similar between treatment groups (NVA237: 57.5\%; PBO: 65.2\%). Serious AEs were reported by $7.5 \%$ of NVA237- vs $9.0 \%$ of PBO-treated patients.

Conclusion NVA237 $50 \mu \mathrm{g}$ once daily was generally safe and well tolerated. Improvements in bronchodilation were rapid, clinically meaningful and maintained for $24 \mathrm{~h}$ throughout the study.

\section{P254 NVA237 ONCE DAILY IMPROVES EXERCISE ENDURANCE IN PATIENTS WITH COPD FROM THE FIRST DOSE: THE GLOW3 TRIAL}

doi:10.1136/thoraxjn|-2011-201054c.254

${ }^{1} \mathrm{D}$ Singh, ${ }^{2} \mathrm{~K}$ Beeh, ${ }^{3} \mathrm{~A}$ Drollmann, ${ }^{3} \mathrm{~L}$ Di Scala, ${ }^{4} \mathrm{R}$ Smith. ${ }^{1}$ University of Manchester, Medicines Evaluation Unit, Manchester, UK; ${ }^{2}$ insaf Respiratory Research Institute, Wiesbaden, Germany; ${ }^{3}$ Novartis Pharma AG, Basel, Switzerland; ${ }^{4}$ Novartis Horsham Research Centre, West Sussex, UK

Introduction The fundamental characteristics of COPD are exertional dyspnoea and exercise limitation, which are associated with 